\title{
Quark jets scattering from a gluon field: From saturation to high $p_{t}$
}

\author{
Jamal Jalilian-Marian \\ Department of Natural Sciences, Baruch College, CUNY, 17 Lexington Avenue, \\ New York, New York 10010, USA \\ and CUNY Graduate Center, 365 Fifth Avenue, New York, New York 10016, USA
}

(Received 18 September 2018; published 30 January 2019)

\begin{abstract}
We continue our studies of possible generalization of the color glass condensate effective theory of high energy QCD to include the high $p_{t}$ (or equivalently large $x$ ) QCD dynamics as proposed in [Phys. Rev. D 96, 074020 (2017)]. Here, we consider scattering of a quark from both the small and large $x$ gluon degrees of freedom in a proton or nucleus target and derive the full scattering amplitude by including the interactions between the small and large $x$ gluons of the target. We thus generalize the standard eikonal approximation for parton scattering, which can now be deflected by a large angle (and therefore have large $p_{t}$ ) and also lose a significant fraction of its longitudinal momentum (unlike the eikonal approximation). The corresponding production cross section can thus serve as the starting point toward the derivation of a general evolution equation that would contain the Dokshitzer-Gribov-Lipatov-Altarelli-Parisi evolution equation at large $Q^{2}$ and the Jalilian-Marian-Iancu-McLerran-Weigert-Leonidov-Kovner evolution equation at small $x$. This amplitude can also be used to construct the quark Feynman propagator, which is the first ingredient needed to generalize the color glass condensate effective theory of high energy QCD to include the high $p_{t}$ dynamics. We outline how it can be used to compute observables in the large $x$ (high $p_{t}$ ) kinematic region where the standard color glass condensate formalism breaks down.
\end{abstract}

DOI: 10.1103/PhysRevD.99.014043

\section{INTRODUCTION}

Twist expansion and the collinear factorization approach [1] to particle production in QCD is a powerful and extremely useful formalism for particle production in high energy hadronic/nuclear collisions at high $p_{t}$. However, it is not expected to be valid at high energy and/or for large nuclei where twist expansion breaks down due to high gluon density effects (gluon saturation). The color glass condensate (CGC) formalism (see Ref. [2] for reviews) is an effective field theory approach to QCD at high energies which relies on the fact that at high energy (or at small $x$ ) a hadron or nucleus wave function contains many gluons, referred to as gluon saturation [3,4], and hence is a dense many-body system which is most efficiently described via semiclassical methods [5]. The most significant aspect of CGC is perhaps the emergence of a dynamical scale, called the saturation scale $Q_{s}$, which grows with energy (or $1 / x$ ) and hence can be semihard. The CGC formalism thus can be used to compute quantities such as gluon multiplicities, etc., which are not amenable to the standard perturbative

Published by the American Physical Society under the terms of the Creative Commons Attribution 4.0 International license. Further distribution of this work must maintain attribution to the author(s) and the published article's title, journal citation, and DOI. Funded by SCOAP . methods. Even though applications of the CGC formalism to hadronic/nuclear processes in a limited range of kinematics at the Relativistic Heavy Ion Collider and the LHC have been quite successful [6], the CGC formalism has its shortcomings; namely it is not valid when one probes large $x$ modes of the target proton or nucleus. In case of particle production in hadronic collisions, this happens when high $p_{t}$ particles are produced since $x$ and $p_{t}$ are kinematically related, $x \sim \frac{p_{t}}{\sqrt{s}}$. This is specially important for particle production in mid rapidity as well as the LHC where, due to the large center-of-mass energy of the collision, a large range of transverse momenta becomes accessible. Furthermore, the large $x$ region will be the dominant part of kinematics covered in the proposed Electron Ion Collider, at least in the earliest stages [7]. Therefore, it is desirable to devise a formalism that not only incorporates the physics of saturation but also has the correct high $p_{t}$ and/or large $x$ physics encoded (see also Ref. [8] for generalized splitting functions and the evolution of gluon Transverse Momentum Dependents from small to large $x$ ).

Generalizing the CGC formalism to include high $p_{t}$ physics would have significant ramifications not only for saturation physics and the quest to determine its domain of applicability, but it would also enable one to describe a wide range of phenomena using the same formalism. For example, saturation physics is commonly employed to provide the initial conditions for the hydrodynamic evolution of the medium, the quark gluon plasma, created in 
high energy heavy ion collisions but is not applicable to jet (radiative) energy loss and the interactions between high $p_{t}$ partons and the produced medium. Elastic energy loss and loss (shift) of rapidity are also not present in the current formulation of saturation physics but must be included in a more general description which could be specially significant for cold matter energy loss and $p_{t}$ broadening.

Toward this goal, we proposed a more general approach in Ref. [9]. We considered a high energy quark scattering from a target proton or nucleus, whereas the quark not only scatters from the small $x$ gluons of the target, represented by a soft color field, but also scatters from the large $x$ gluons in the target. We resummed the multiple scatterings of the quark from the soft classical fields to all orders in the number of soft scatterings but kept only the first scattering from the large $x$ modes. However, we did not consider the large $x$ modes themselves scattering from the small $x$ gluons. Here, we continue this approach and proceed to include interactions between the large $x$ gluons and the soft background field representing small $x$ gluons of the target.

We start with a brief overview of the approximations used in high energy (eikonal) scattering and how it is used in saturation physics when viewed in the target rest frame. We then give a brief summary of the approximations and the methods used in our approach in Ref. [9]. We again consider scattering of a quark from a proton or nucleus target including both small and large $x$ gluon modes. We then proceed to calculate and resum multiple interactions between the small $x$ color fields and the large $x$ gluons in the target. We then briefly outline how the calculated scattering amplitude may be used to extract the quark propagator in this more general setting and how the quark propagator may be used to compute physical observables in the full range of $x$ (and/or $p_{t}$ ).

\section{EIKONAL APPROXIMATION, MULTIPLE SCATTERING AT SMALL $x$ AND BEYOND}

Here, we remind the reader of the approximations involved in high energy (eikonal) scattering. As this is standard and already covered in detail in Ref. [9], we will be brief here. We define the light cone coordinates as

$$
x^{+} \equiv \frac{t+z}{\sqrt{2}}, \quad x^{-} \equiv \frac{t-z}{\sqrt{2}}
$$

and similarly for momenta and fields. The small $x$ gluons of the target are modeled as a soft color (background) field $S_{a}^{\mu}(x)$. One can either work in the frame in which both the projectile and the target are moving fast or work in the frame in which the projectile quark is fast and the target is at rest. In either frame, the projectile quark moving to the right (along the $x^{+}$direction to be specific) will have a large $p^{+}$component of momentum and will couple to the conjugate component of the target color field $S^{-}$. Furthermore, the target color field is independent of $x^{-}$ so that $S_{a}^{-}=S_{a}^{-}\left(x^{+}, x_{t}\right)$. We also define a lightlike vector,

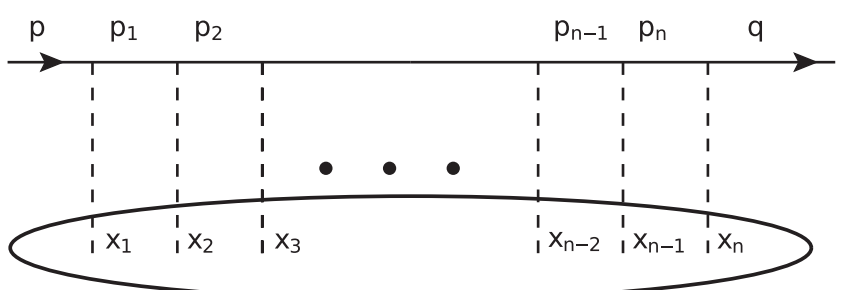

FIG. 1. Multiple soft scatterings from the color field of the target.

$$
n^{\mu}=\left(n^{+}=0, n^{-}=1, n_{t}=0\right),
$$

with $n^{2}=0$, which can be used to extract the Lorentz index of the soft color field and express it as $S_{a}^{-} \equiv n^{-} S_{a}\left(x^{+}, x_{t}\right)$ so that $\delta_{a}=S_{s}^{\mu} \gamma_{\mu}=\not h S_{a}$, which will help keep the expressions compact.

The $n$th order of the scattering of a quark, with momentum $p$, from the color field of the target is depicted in Fig. 1 (target is shown as an ellipse) where $x_{i}$ label the coordinate positions of the field $S^{-}$in the target (one should think of this as the projectile quark multiply scattering while going right through the target so that there are no propagators between the quark line and the target field). Diagrams of this type resum into a path-ordered infinite Wilson line, provided one neglects the transverse momenta of the intermediate quark lines and the phases one picks up after integrating over the $p_{i}^{-}$of each intermediate quark propagator $p_{i}^{\mu}$. The integration over the minus component of the intermediate propagators forces a path ordering such that the scattering is sequential along the longitudinal direction, i.e., $x_{i}^{+}>x_{i-1}^{+}$ and so on (see Refs. [9-11] for details).

The scattering amplitude can then be written as

$$
\begin{aligned}
& i \mathcal{M}_{\text {eikonal }}(p, q) \\
& \quad=2 \pi \delta\left(p^{+}-q^{+}\right) \bar{u}(q) \not h \int d^{2} x_{t} e^{-i\left(q_{t}-p_{t}\right) \cdot x_{t}}\left[V\left(x_{t}\right)-1\right] u(p),
\end{aligned}
$$

where the infinite Wilson line $V\left(x_{t}\right)$ is defined as

$$
V\left(x_{t}\right) \equiv \hat{P} \exp \left\{i g \int_{-\infty}^{+\infty} d x^{+} S_{a}^{-}\left(x^{+}, x_{t}\right) t_{a}\right\}
$$

and depicted in Fig. 2. This infinite Wilson line resums multiple scatterings of a high energy quark (moving along the positive $z$ axis) on a soft background color field to all orders in the soft field $\left[i g S_{a} t_{a}\right]^{n}$. Due to the eikonal approximation (see also Ref. [12] in which the first energy suppressed terms are investigated), the transverse position of the quark does not change during the scattering; i.e., the projectile quark does not get a significant deflection (small angle scattering). Color matrices $t_{a}$ are in the fundamental representation, and soft color field $S^{-}$represents small $x$ gluon modes of the target. 


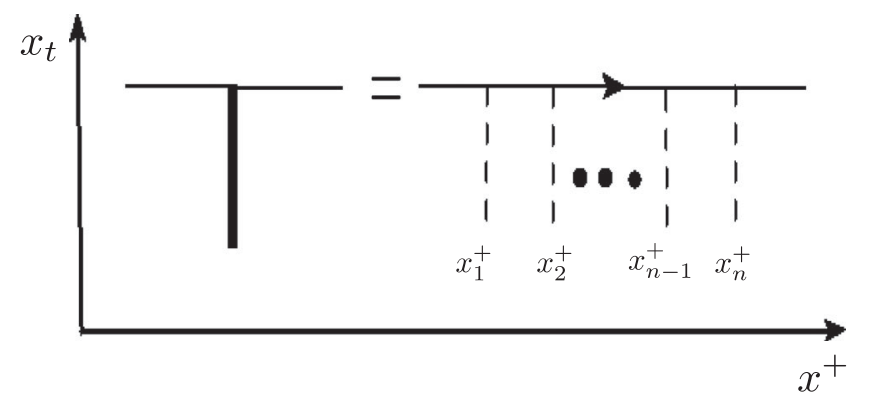

FIG. 2. A Wilson line representing eikonal scattering of a high energy quark.

In Ref. [9], we went beyond eikonal approximation by including scattering from a large $x$ gluon field denoted $A^{\mu}(x)$, which, unlike the soft field $S^{-}$, carries large longitudinal momentum and can therefore cause a large deflection of the projectile quark. Due to the possibility of this large angle deflection (so that the final state quark has a large transverse momentum), it was necessary to introduce a rotated frame, denoted bar-ed frame, in which the scattered quark is moving along a new longitudinal direction $\bar{z}$. The bar-ed coordinates $\bar{x}, \bar{y}, \bar{z}$ are related to the original $x, y, z$ coordinates (the projectile quark is moving along the $z$ direction) via the rotation matrix $\mathcal{O}$ in 3 dimensions,

$$
\left(\begin{array}{l}
\bar{x} \\
\bar{y} \\
\bar{z}
\end{array}\right)=\mathcal{O}\left(\begin{array}{l}
x \\
y \\
z
\end{array}\right) .
$$

The elements of this three-dimensional rotation matrix $\mathcal{O}$ are expressed in terms of the 3-momentum of the scattered quark. We also defined a new lightlike vector $\bar{n}^{\mu}=\left(\bar{n}^{+}=0, \bar{n}^{-}=1, \bar{n}_{t}=0\right)$, which projects out the plus component in the new frame, so that $\bar{n} \cdot \bar{p}=\bar{p}^{+}$. We then managed to resum all the multiple scatterings of the projectile quark from the soft field and one scattering from the large $x$ (sometimes referred to as the hard field, where "hard" refers to a large longitudinal momentum). This is shown in Fig. 3 (the target is not explicitly drawn), and diagrams of this type resum into [9]

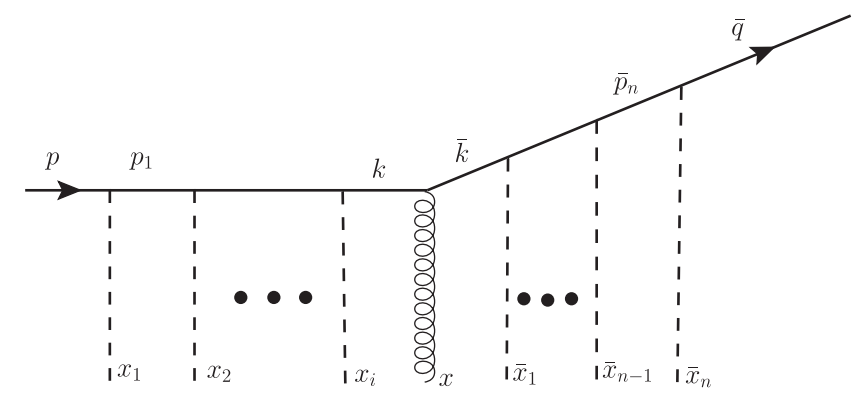

FIG. 3. Multiple soft scatterings before and after a hard one at $x$.

$$
\begin{aligned}
i \mathcal{M}_{1}= & \int d^{4} x d^{2} z_{t} d^{2} \bar{z}_{t} \int \frac{d^{2} k_{t}}{(2 \pi)^{2}} \frac{d^{2} \bar{k}_{t}}{(2 \pi)^{2}} e^{i(\bar{k}-k) x} \\
& \times e^{-i\left(\bar{q}_{t}-\bar{k}_{t}\right) \cdot \bar{z}_{t}} e^{-i\left(k_{t}-p_{t}\right) \cdot z_{t}} \bar{u}(\bar{q}) \\
& \times\left[\bar{V}_{A P}\left(x^{+}, \bar{z}_{t}\right) \not h \frac{\bar{k}}{2 \bar{k}^{+}}[i g A(x)] \frac{\not k}{2 k^{+}} \not h V_{A P}\left(z_{t}, x^{+}\right)\right] u(p)
\end{aligned}
$$

with $k^{+}=p^{+}, k^{-}=\frac{k_{t}^{2}}{2 k^{+}}, \bar{k}^{+}=\bar{q}^{+}, \bar{k}^{-}=\frac{\bar{k}_{t}^{2}}{2 \bar{k}^{+}}$, and the semiinfinite, anti-path-ordered Wilson lines in the fundamental representation are now defined as ${ }^{1}$

$$
\bar{V}_{A P}\left(x^{+}, \bar{z}_{t}\right) \equiv \hat{P} \exp \left\{i g \int_{x^{+}}^{+\infty} d \bar{z}^{+} \bar{S}_{a}^{-}\left(\bar{z}_{t}, \bar{z}^{+}\right) t_{a}\right\}
$$

and

$$
V_{A P}\left(z_{t}, x^{+}\right) \equiv \hat{P} \exp \left\{i g \int_{-\infty}^{x^{+}} d z^{+} S_{a}^{-}\left(z_{t}, z^{+}\right) t_{a}\right\},
$$

where anti-path-ordering (AP) in the amplitude means fields with the largest argument appear to the left.

\section{A. Multiple scatterings of the large $x$ gluon}

We now proceed to consider interactions of the hard (large $x$ ) gluon with the soft background field. First, let us consider the case in which only the hard gluon interacts with the soft field but not the initial or final state quark, as shown in Fig. 4. The amplitude can be written as

$$
\begin{aligned}
i \mathcal{M}= & \int \frac{d^{4} k}{(2 \pi)^{4}} d^{4} x d^{4} x_{1} e^{i(\bar{q}-p-k) x_{1}} e^{i k x} \bar{u}(\bar{q})\left(i g \gamma^{\mu} t^{a}\right) u(p) \\
& \times G_{\mu \nu}^{a b}(p-\bar{q}) V_{b c d}^{\nu \lambda \rho}(p-\bar{q}, k, \bar{q}-p-k) \\
& \times A_{\lambda}^{c}(x)\left[i g S_{\rho}^{d}\left(x_{1}\right)\right]
\end{aligned}
$$

where the free gluon propagator is

$$
G_{a b}^{\mu \nu}(l)=\frac{i \delta_{a b}}{l^{2}+i \epsilon}\left[-g^{\mu \nu}+\frac{n^{\mu} l^{\nu}+l^{\mu} n^{\nu}}{n \cdot l}\right]
$$

and the triple gluon vertex is

$$
\begin{aligned}
& V_{\nu \lambda \rho}^{b c d}\left(l_{1}, l_{2}, l_{3}\right) \\
& \quad=g f^{b c d}\left[\left(l_{2}-l_{3}\right)_{\nu} g_{\lambda \rho}+\left(l_{3}-l_{1}\right)_{\lambda} g_{\rho \nu}+\left(l_{1}-l_{2}\right)_{\rho} g_{\nu \lambda}\right] .
\end{aligned}
$$

The large $x$ gluon field is denoted $A\left(x^{+}, x^{-}, x_{t}\right)$, while the soft field is $S_{d}^{-}\left(x_{1}^{+}, x_{1 t}\right)=n^{-} S_{d}\left(x_{1}^{+}, x_{1 t}\right)$. It is straightforward to simplify the Lorentz structure of the amplitude by repeated use of the gauge condition $n \cdot A=0$ as well as the

\footnotetext{
${ }^{1}$ See Ref. [9] regarding the use of path- vs anti-path-ordered Wilson lines in propagators vs amplitudes.
} 


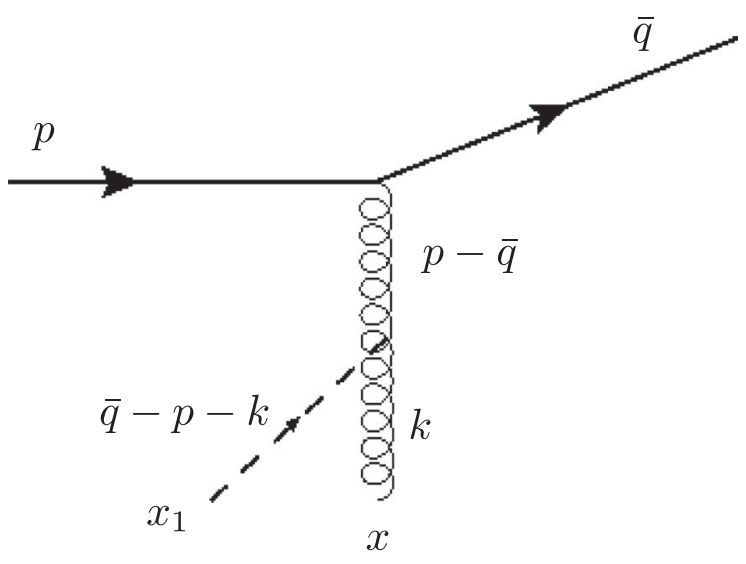

FIG. 4. One soft scattering of the large $x$ gluon.

null condition $n^{2}=0$ and the fact that the soft field has only a - component and therefore is proportional to $n^{\mu}$. We get

$$
\begin{aligned}
i \mathcal{M}= & f_{a c d} \int \frac{d^{4} k}{(2 \pi)^{4}} d^{4} x d^{4} x_{1} e^{i(\bar{q}-p-k) x_{1}} \\
& \times e^{i k x} \bar{u}(\bar{q})\left(i g \gamma^{\mu} t^{a}\right) u(p) A_{\lambda}^{c}(x)\left[i g S^{d}\left(x_{1}\right)\right] \\
& \times \frac{1}{(p-\bar{q})^{2}+i \epsilon}\left[-g_{\lambda}^{\mu} n \cdot(p-\bar{q}-k)\right. \\
& \left.+n^{\mu}\left[p_{\lambda}-\bar{q}_{\lambda}\left(1-\frac{n \cdot k}{n \cdot(p-\bar{q})}\right)\right]\right] .
\end{aligned}
$$

As before, the soft field $S$ is independent of the $x_{1}^{-}$ coordinate, which allows us to do the integration over the $x_{1}^{-}$coordinate, which gives $\delta\left(\bar{q}^{+}-p^{+}-k^{+}\right)$, which in turn is used to do the $k^{+}$integration setting $k^{+}=\bar{q}^{+}-p^{+}$ upon integrating $k^{+}$. We also note that the only $k$ dependence left is in the phase factors since $n \cdot k$ kills all the other possible $k$ dependent terms. Then, one can carry out the $k_{t}$ and $k^{-}$integrations which lead to delta functions $\delta^{2}\left(x_{1 t}-x_{t}\right) \delta\left(x_{1}^{+}-x^{+}\right)$, which are then used to perform the remaining integrations over $x_{1}^{+}, x_{1 t}$ setting $x_{1 t}=x_{t}$ and $x_{1}^{+}=x^{+}$. In other words, the soft field $S$ and the large $x$ (hard) field $A$ are at the same space coordinate except that the soft field $S$ does not depend on $x^{-}$, unlike the hard field $A$. After performing the above integrations, we are left with

$$
\begin{aligned}
i \mathcal{M}= & 2 f_{\text {acd }} \int d^{4} x e^{i(\bar{q}-p) x} \bar{u}(\bar{q}) \\
& \times \frac{\left[\not h(p-\bar{q}) \cdot A_{c}(x)-\not A_{c}(x) n \cdot(p-\bar{q})\right]}{(p-\bar{q})^{2}}\left(i g t^{a}\right) u(p) \\
& \times\left[i g S^{d}\left(x^{+}, x_{t}\right)\right]
\end{aligned}
$$

with the most important point being that the soft and hard field are at the same point. We now go ahead and consider

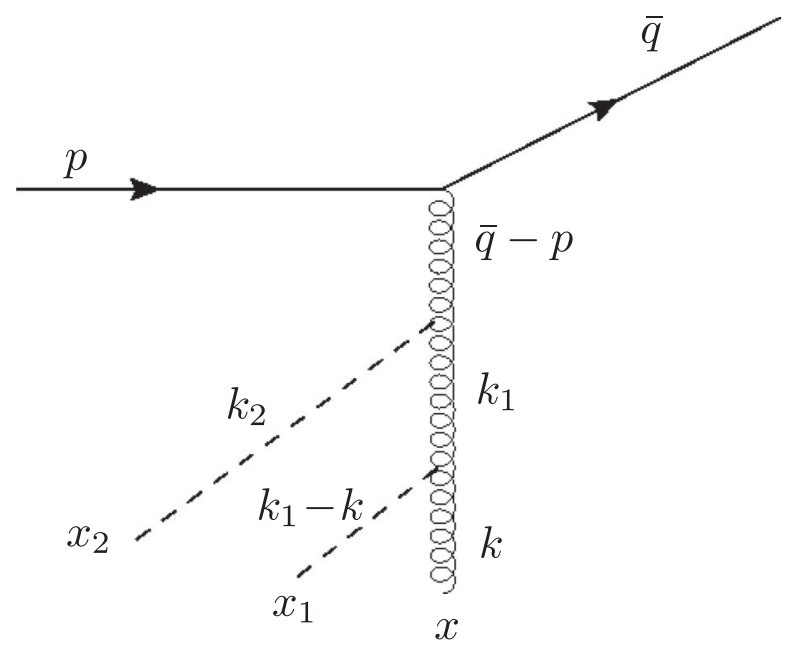

FIG. 5. Two soft scatterings of the large $x$ gluon.

one more soft scattering of the hard gluon as shown in Fig. 5. As before we use the gauge and null vector conditions to simplify the Lorentz structure. We then perform the integration over $k$ which sets the fields $A(x)$ and $S\left(x_{1}\right)$ at the same point $\left(x_{1}=x\right)$ after which there is an integration over $k_{1}$ left. Let us now consider the $k_{1}^{-}$ integration,

$$
I \equiv \int \frac{d k_{1}^{-}}{(2 \pi)} \frac{e^{i k_{1}^{-}\left(x^{+}-x_{2}^{+}\right)}}{2\left(\bar{q}^{+}-p^{+}\right)\left[k_{1}^{-}-\frac{k_{11^{+}}^{2}-i \epsilon}{2\left(\bar{q}^{+}-p^{+}\right)}\right]} .
$$

This integration can be done using the standard contour integration techniques realizing that the $k_{1}^{-}$pole is always below the real axis since $p^{+}>\bar{q}^{+}$. This integral is then

$$
I \sim \frac{i \theta\left(x^{+}-x_{2}^{+}\right)}{2 n \cdot(\bar{q}-p)} e^{i \frac{k_{1 t}^{2}}{2 n \cdot(\bar{q}-p)}\left(x^{+}-x_{2}^{+}\right)} .
$$

The most essential point here is the reappearance of the theta function $\theta\left(x^{+}-x_{2}^{+}\right)$, which forces a path ordering of the soft scatterings. This is not surprising since the soft multiple scatterings are eikonal. The rest of the analysis goes through as before, and we get

$$
\begin{aligned}
i \mathcal{M}= & 2 f_{a b c} f_{c d e} \int d^{4} x d x_{2}^{+} \theta\left(x^{+}-x_{2}^{+}\right) e^{i\left(\bar{q}^{+}-p^{+}\right) x^{-}-i\left(\bar{q}_{t}-p_{t}\right) \cdot x_{t}} \\
& \times \bar{u}(\bar{q}) \frac{\left[\not h(p-\bar{q}) \cdot A_{e}(x)-\not A_{c}(x) n \cdot(p-\bar{q})\right]}{(p-\bar{q})^{2}}\left(i g t^{a}\right) u(p) \\
& \times\left[i g S_{d}\left(x^{+}, x_{t}\right)\right]\left[i g S_{b}\left(x_{2}^{+}, x_{t}\right)\right] .
\end{aligned}
$$

We now consider a third soft scattering of the large $x$ gluon, which should make the anticipated exponentiation of the soft multiple scattering more clear. This is shown in Fig. 6 with the amplitude given by 


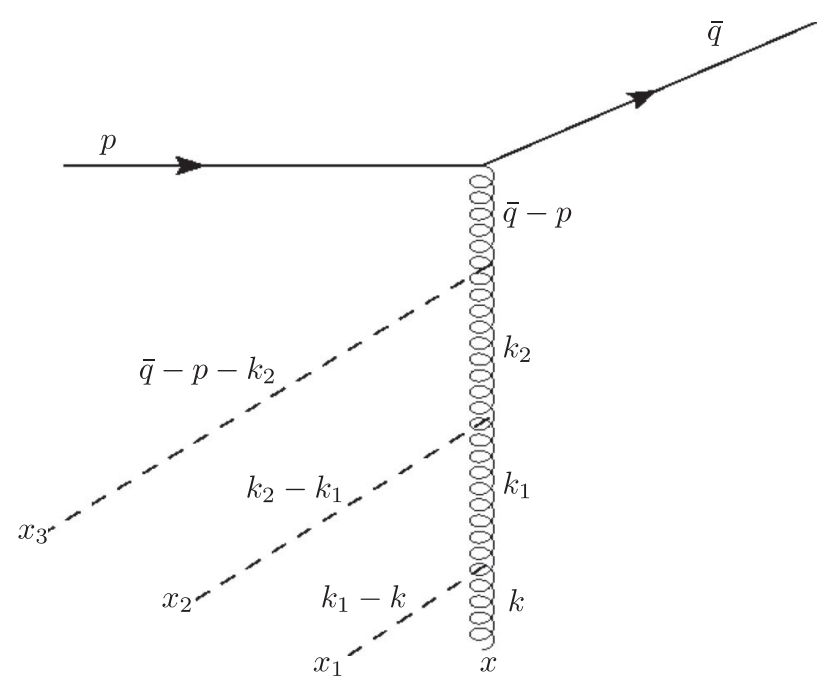

FIG. 6. Three soft scatterings of the large $x$ gluon.

$$
\begin{aligned}
i \mathcal{M}= & \int d^{4} x d^{4} x_{1} d^{4} x_{2} d^{4} x_{3} \frac{d^{4} k}{(2 \pi)^{4}} \frac{d^{4} k_{1}}{(2 \pi)^{4}} \frac{d^{4} k_{2}}{(2 \pi)^{4}} \\
& \times e^{i k x} e^{i\left(k_{1}-k\right) x_{1}} e^{i\left(k_{2}-k_{1}\right) x_{2}} e^{i\left(\bar{q}-p-k_{2}\right) x_{3}} \\
& \times \bar{u}(\bar{q}) \gamma_{\mu}\left(i g t^{a}\right) \Gamma_{a}^{\mu} u(p)
\end{aligned}
$$

with

$$
\begin{aligned}
\Gamma_{a}^{\mu} \equiv & G^{\mu \nu}(\bar{q}-p) V_{\nu \rho \lambda}^{a b c}\left(p-\bar{q}, \bar{q}-p-k_{2}, k_{2}\right) \\
& \times G^{\lambda \delta}\left(k_{2}\right) V_{\delta \gamma \alpha}^{c d e}\left(-k_{2}, k_{2}-k_{1}, k_{1}\right) \\
& \times G^{\alpha \beta}\left(k_{1}\right) V_{\beta \sigma \epsilon}^{e g f}\left(-k_{1}, k_{1}-k, k\right) A_{f}^{\epsilon}(x) \\
& \times n^{\sigma} S_{g}\left(x_{1}\right) n^{\delta} S_{d}\left(x_{2}\right) n^{\rho} S_{b}\left(x_{3}\right)
\end{aligned}
$$

We now proceed as before and perform the various integrations involved using the fact that the soft field $S(z)$ does not depend on the $z^{-}$coordinate, which leads to conservation of the plus component of momentum at the soft vertex. As before, the only dependence on $k^{-}, k_{t}$ is in the exponentials, integration over which eventually sets $x_{1}^{+}=x^{+}$and $x_{1 t}=x_{t}$. The integrations over the minus components of internal momenta $k_{1}^{-}, k_{2}^{-}$can be done via contour integration techniques and lead to path ordering of the soft scatterings as before enforced by $\theta\left(x^{+}-x_{2}^{+}\right) \theta\left(x_{2}^{+}-x_{3}^{+}\right)$. Performing the various Lorentz contractions and using the gauge condition as well as $n^{2}=0$, the amplitude can be simplified to

$$
\begin{aligned}
i \mathcal{M}= & \frac{2(i)^{2}}{(\bar{q}-p)^{2}} f^{a b c} f^{c d e} f^{e g f} \\
& \times \int d^{4} x d x_{2}^{+} d x_{3}^{+} \theta\left(x^{+}-x_{2}^{+}\right) \theta\left(x_{2}^{+}-x_{3}^{+}\right) \\
& \times \bar{u}(\bar{q})\left(i g t^{a}\right)\left[n \cdot(p-\bar{q}) A_{f}(x)-(p-\bar{q}) \cdot A_{f}(x) \not h\right] u(p) \\
& \times\left[i g S_{g}\left(x^{+}, x_{t}\right)\right]\left[i g S_{d}\left(x_{2}^{+}, x_{t}\right)\right]\left[i g S_{b}\left(x_{3}^{+}, x_{t}\right)\right] \\
& \times e^{i\left(\bar{q}^{+}-p^{+}\right) x^{-}-i\left(\bar{q}_{t}-p_{t}\right) \cdot x_{t}} .
\end{aligned}
$$

By considering the derivative of the path-ordered Wilson line $U$, we see that the calculated terms in (12), (15), (18) correspond to the first three nontrivial terms in the expansion of the Wilson line as

$$
\begin{aligned}
\partial_{x^{+}}\left[U_{A P}^{\dagger}\left(x_{t}, x^{+}\right)\right]^{a b}= & \left(i f^{b c a}\right)\left[i g S_{c}\left(x^{+}, x_{t}\right)\right] \\
& +\left(i f^{b c e}\right)\left(i f^{e d a}\right) \int d x_{1}^{+} \theta\left(x^{+}-x_{1}^{+}\right)\left[\left[i g S_{c}\left(x^{+}, x_{t}\right)\right]\left[i g S_{d}\left(x_{1}^{+}, x_{t}\right)\right]\right. \\
& +\left(i f^{b c h}\right)\left(i f^{g d f}\right)\left(i f^{f e a}\right) \int d x_{1}^{+} d x_{2}^{+} \theta\left(x^{+}-x_{1}^{+}\right) \theta\left(x_{1}^{+}-x_{2}^{+}\right) \\
& \times\left[[ i g S _ { c } ( x ^ { + } , x _ { t } ) ] [ i g S _ { d } ( x _ { 1 } ^ { + } , x _ { t } ) ] \left[\left[i g S_{c}\left(x_{2}^{+}, x_{t}\right)\right]+\cdots \cdots,\right.\right.
\end{aligned}
$$

and this can be generalized to all orders and allows one to resum all the soft scatterings of the hard gluon and write it as

$$
\begin{aligned}
i \mathcal{M}_{2}= & \frac{2 i}{(p-\bar{q})^{2}} \int d^{4} x e^{i\left(\bar{q}^{+}-p^{+}\right) x^{-}-i\left(\bar{q}_{t}-p_{t}\right) \cdot x_{t}} \\
& \times \bar{u}(\bar{q})\left[\left(i g t^{a}\right)\left[\partial_{x^{+}} U_{A P}^{\dagger}\left(x_{t}, x^{+}\right)\right]^{a b}\right. \\
& \left.\times\left[n \cdot(p-\bar{q}) \mathbb{A}_{b}(x)-(p-\bar{q}) \cdot A_{b}(x) \not h\right]\right] u(p),
\end{aligned}
$$

where the derivative acts on the + coordinate of the adjoint Wilson line (anti-path-ordered in the amplitude) and arises from the fact that one can write the soft field at the last scattering point as a derivative on the Wilson line. This amplitude is symbolically shown in Fig. 7, in which the thick solid line attached to the hard gluon line depicts a semiinfinite (and anti-path-ordered) Wilson line in the adjoint representation [analog of Eq. (8)]. ${ }^{2}$ It should be noted that we have dropped terms of the form $e^{i\left(\bar{q}^{-}-p^{-}\right) x_{n}^{+}}$, where $x_{n}^{+}$is the location of the last soft scattering of the large gluon field before the quark vertex. This is best understood in the infinite momentum frame in which the soft color field is a shock wave at $x_{i}^{+}=0$ so that the exponential factor is just unity.

\footnotetext{
${ }^{2}$ Recall that adjoint representation is real so that $\left[U^{\dagger}\right]^{a b}=U^{b a}$.
} 


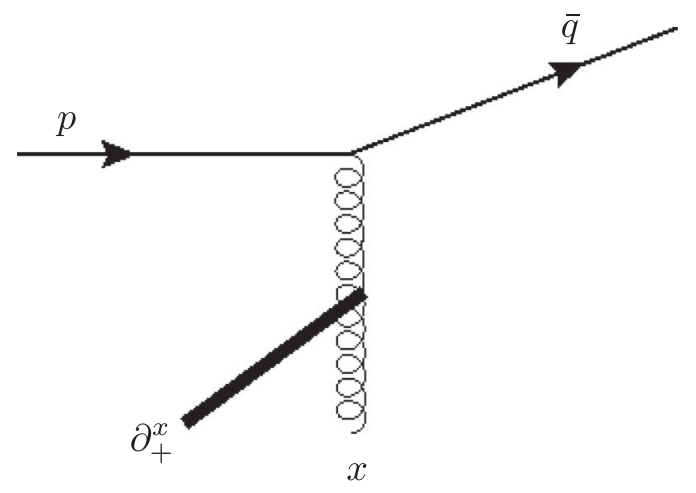

FIG. 7. Multiple soft scatterings of the large $x$ gluon.

Finally, we note that this amplitude vanishes in the soft (eikonal) limit, i.e., when $\bar{q}^{+} \rightarrow p^{+}$and $A^{\mu} \rightarrow S^{-}$.

\section{B. Multiple scatterings of the large $x$ gluon and the final state quark}

We now consider the case in which both the large $x$ gluon and the final state quark multiply scatter from the soft background field. The first diagram not included so far is when both the large $x$ gluon and the final state quark scatter once as shown in Fig. 8. This scattering amplitude is given by

$$
\begin{aligned}
i \mathcal{M}= & \int \frac{d^{4} k}{(2 \pi)^{4}} \frac{d^{4} \bar{p}_{1}}{(2 \pi)^{4}} d^{4} x d^{4} x_{1} d^{4} \bar{x}_{1} \\
& \times e^{i\left(\bar{q}-\bar{p}_{1}\right) \bar{x}_{1}} e^{-i k x} e^{-i\left(p-\bar{p}_{1}-k\right) x_{1}} \bar{u}(\bar{q}) \not{h}\left[i g \bar{S}\left(\bar{x}_{1}\right)\right] \\
& \times \frac{i \not_{1}}{\bar{p}_{1}^{2}+i \epsilon}\left(i g \gamma^{\mu} t^{a}\right) u(p) G_{\mu \nu}\left(p-\bar{p}_{1}\right) \\
& \times V_{a b c}^{\nu \lambda \rho}\left(p-\bar{p}_{1},-k, \bar{p}_{1}+k-p\right) A_{\lambda}^{b}(x)\left[i g n_{\rho} S^{c}\left(x_{1}\right)\right] .
\end{aligned}
$$

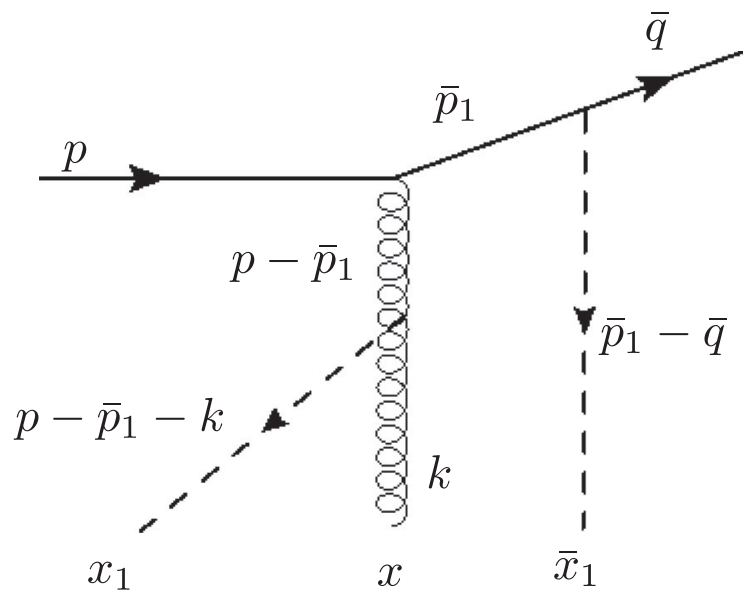

FIG. 8. Both the large $x$ gluon and the final state quark scattering once.
Many of the steps involved in simplifying this expression are identical to the previous ones; for example, we use the fact that the soft field $S^{-}\left(x_{i}\right)$ is independent of the $x_{i}^{-}$ coordinate to perform the integration over the minus components of their coordinates, leading to delta functions relating the + components of the momenta. The Lorentz structure can also be simplified as before by repeated use of the gauge condition as well as the fact that $n^{2}=\bar{n}^{2}=0$ and that the soft fields (in their respective frames) have only minus components, which allows extraction of their Lorentz index by the use of the lightlike vectors $n$ and $\bar{n}$ so that $S^{-}=n^{-} S$ and $\bar{S}^{-}=\bar{n}^{-} \bar{S}$. As in the case of only the hard gluon scattering we just considered, one gets $x_{1}^{+}=x^{+}$ and $x_{1 t}=x_{t}$. The next step is to consider integration over $\bar{p}_{1}^{-}$momentum of the intermediate quark line which is

$$
I \sim \int \frac{d \bar{p}_{1}^{-}}{(2 \pi)} \frac{e^{-i \bar{p}_{1}^{-}\left(\bar{x}_{1}^{+}-x^{+}\right)}}{\left[\bar{p}_{1}^{-}-\frac{\bar{p}_{1 t^{-}-i \epsilon}}{2 \bar{q}^{+}}\right]\left[\bar{p}_{1}^{-}-p^{-}-\frac{\left(\bar{p}_{1 t}-p_{t}\right)^{2}-i \epsilon}{2\left(\bar{q}^{+}-p^{+}\right)}\right]},
$$

keeping in mind that both $p^{+}, \bar{q}^{+}>0$ and that $p^{+}-\bar{q}^{+}>0$ we see that the integral above has two poles which are on the opposite side of the real axis. This is completely different from the eikonal scattering in which the intermediate quark propagators have poles which are all on the same side of the real axis, which leads to path ordering along the + direction. This integral can be evaluated using the standard contour integration techniques and gives

$$
\begin{aligned}
I \sim & {\left[\theta\left(\bar{x}_{1}^{+}-x^{+}\right) e^{-i \frac{\bar{p}_{1 t}^{2}}{2 \bar{q}^{+}}\left(\bar{x}_{1}^{+}-x^{+}\right)}\right.} \\
& \left.+\theta\left(x^{+}-\bar{x}_{1}^{+}\right) e^{-i\left[p^{-}+\frac{\left(\bar{p}_{1}-p_{t}\right)^{2}}{2\left(\bar{q}^{+}-p^{+}\right)}\right]\left(\bar{x}_{1}^{+}-x^{+}\right)}\right] .
\end{aligned}
$$

To proceed further and to stay consistent with the approximations made for strict eikonal scattering in which one neglects terms of the order $\frac{p_{t}}{p^{+}}$, we will ignore the phase factors above. We then see that the two different path orderings corresponding to the two theta functions add to unity and path ordering disappears. This can be understood as the following: integration over any of the poles forces the other propagator to go off shell and to become spacelike, in which case there is no absolute ordering between the interaction vertices at $x_{1}^{+}=x^{+}$and $\bar{x}_{1}^{+}$. However, if we consider further soft scatterings of the hard gluon and the final state quark, they will be path ordered with respect to $x^{+}$and $\bar{x}_{1}^{+}$respectively. This is straightforward but tedious, and we will just quote the final result as the calculations proceeds as earlier and there are no further subtle points. Resumming all the soft scatterings of the hard gluon and the final state quark then gives 


$$
\begin{aligned}
i \mathcal{M}_{3}= & -2 i \int d^{4} x d^{2} \bar{x}_{t} d \bar{x}^{+} \frac{d^{2} \bar{p}_{1 t}}{(2 \pi)^{2}} e^{i\left(\bar{q}^{+}-p^{+}\right) x^{-}} e^{-i\left(\bar{p}_{1 t}-p_{t}\right) \cdot x_{t}} e^{-i\left(\bar{q}_{t}-\bar{p}_{1 t}\right) \cdot \bar{x}_{t}} \\
& \times \bar{u}(\bar{q})\left[\left[\partial_{\bar{x}^{+}} \bar{V}_{A P}\left(\bar{x}^{+}, \bar{x}_{t}\right)\right] \bar{h} \bar{p}_{1}\left(i g t^{a}\right)\left[\partial_{x^{+}} U_{A P}^{\dagger}\left(x_{t}, x^{+}\right)\right]^{a b} \frac{\left[n \cdot(p-\bar{q}) A^{b}(x)-\left(p-\bar{p}_{1}\right) \cdot A^{b}(x) \not h\right]}{\left[2 n \cdot \bar{q} 2 n \cdot(p-\bar{q}) p^{-}-2 n \cdot(p-\bar{q}) \bar{p}_{1 t}^{2}-2 n \cdot \bar{q}\left(\bar{p}_{1 t}-p_{t}\right)^{2}\right]}\right] \\
& \times u(p)
\end{aligned}
$$

and is depicted in Fig. 9 below, in which the thick solid lines denote semi-infinite (and anti-path-ordered) Wilson lines in fundamental (attached to the final state quark line) and adjoint (attached to the hard gluon line) representations. Also, we have $\bar{p}_{1}^{+}=\bar{q}^{+}$. We again note that this amplitude also vanishes in the soft limit.

\section{Multiple scatterings of the initial state quark and the large $x$ gluon}

We now consider the next class of diagrams in which both the large $x$ gluon and the initial state quark scatter from the soft background field. The lowest-order diagram, not included so far, is shown in Fig. 10. This amplitude can be written as

$$
\begin{aligned}
i \mathcal{M}= & \int \frac{d^{4} k}{(2 \pi)^{4}} \frac{d^{4} p_{1}}{(2 \pi)^{4}} d^{4} x d^{4} x_{1} d^{4} x_{2} e^{i\left(p_{1}-p\right) x_{1}} e^{-i k x} e^{i\left(k+\bar{q}-p_{1}\right) x_{2}} \\
& \times\left[\bar{u}(\bar{q})\left(i g \gamma^{\mu} t^{a}\right) \frac{i \not p_{1}}{p_{1}^{2}+i \epsilon} \not h\left[i g S\left(x_{1}\right)\right] u(p)\right] G_{\mu \nu}\left(p_{1}-\bar{q}\right) \\
& \times V_{a c d}^{\nu \lambda \rho}\left(p_{1}-\bar{q},-k, k+\bar{q}-p_{1}\right) A_{\lambda}^{c}(x)\left[i g n_{\rho} S^{d}\left(x_{2}\right)\right] .
\end{aligned}
$$

Again, most of the steps are identical to before; one integrates over the - component of the soft fields coordinates, eventually setting some + components of momenta equal to each other. Lorentz structure is simplified using the gauge condition and the null vector $n^{2}=0$ as well as extracting the Lorentz index of the soft field $S^{-}=n^{\mu} S$. Integration over $k$ again sets $x_{2}^{+}=x^{+}$. The crucial step now is the integration over $p_{1}^{-}$, which we focus on,

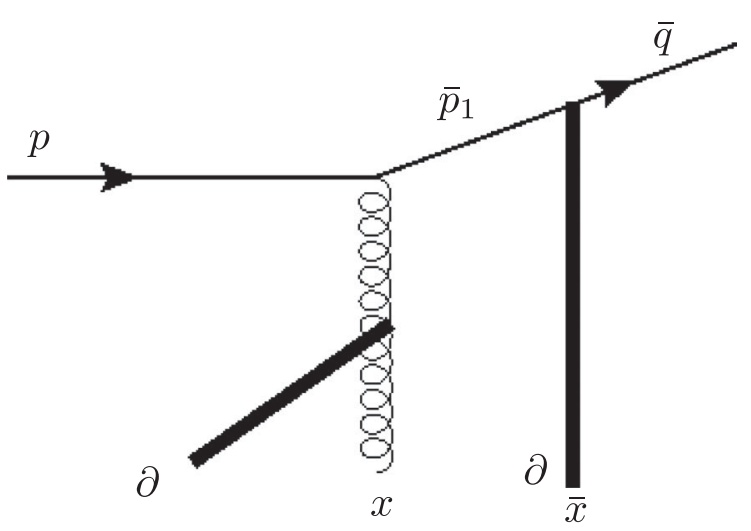

FIG. 9. Soft scatterings of the final state quark and the large $x$ gluon.

$$
I \sim \int \frac{d p_{1}^{-}}{(2 \pi)} \frac{e^{-i p_{1}^{-}\left(x_{1}^{+}-x^{+}\right)}}{\left[p_{1}^{-}-\frac{p_{1 t}^{2}-i \epsilon}{2 p^{+}}\right]\left[p_{1}^{-}-\bar{q}^{-}-\frac{\left(p_{1 t}-\bar{q}_{t}\right)^{2}-i \epsilon}{2\left(p^{+}-\bar{q}^{+}\right)}\right]} .
$$

The two poles are now both below the real axis so that the integration gives a nonzero value only if $x^{+}>x_{1}^{+}$, we get

$I \sim \theta\left(x^{+}-x_{1}^{+}\right)\left[e^{i \frac{p_{1 t}^{2}}{2 p^{+}}\left(x_{1}^{+}-x^{+}\right)}-e^{i\left[\bar{q}^{-}+\frac{\left(p_{1}-\bar{q}_{t}\right)^{2}}{2\left(p^{+}-\bar{q}^{+}\right)}\right]\left(x_{1}^{+}-x^{+}\right)}\right]$.

We note that, unlike in the case of scattering from the large $x$ gluon and the final state quark, the relative sign between the two phase factors is negative (recall that the poles were on the opposite side in that case). Hence, ignoring the phases again consistent with strict eikonal approximation, one gets a cancellation between the two terms so that $I=0$; therefore, this amplitude identically vanishes. It is straightforward to include any number of further soft scatterings from the initial or final state quark or the large $x$ gluon. However, it can be shown these further scatterings do not affect this null result. Therefore, we conclude that one cannot have simultaneous soft scatterings from the initial state quark and the large $x$ gluon. There are no other diagrams to consider; therefore, this completes our derivation of the amplitude for scattering of a quark from the small and large $x$ gluon fields of the target.

The result for the full scattering amplitude at all $x$ (or any $p_{t}$ ) can thus be written as

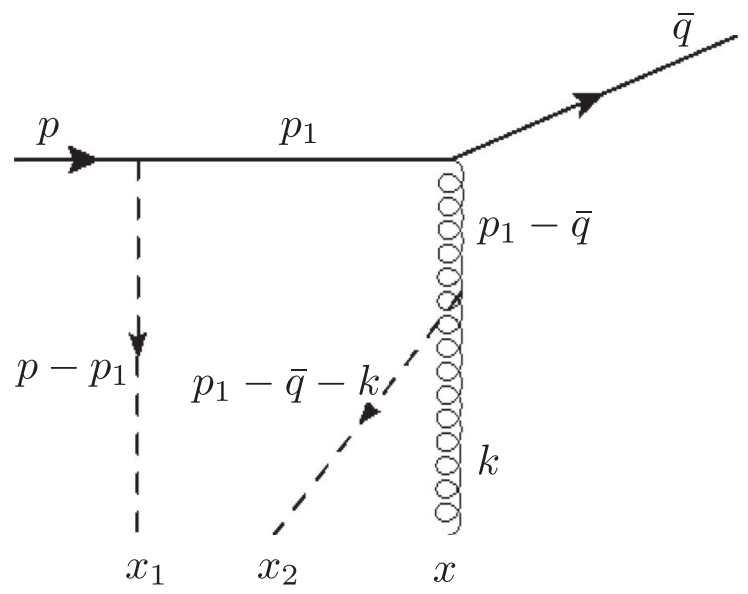

FIG. 10. Soft scatterings of the initial state quark and the large $x$ gluon. 


$$
i \mathcal{M}=i \mathcal{M}_{\text {eikonal }}+i \mathcal{M}_{1}+i \mathcal{M}_{2}+i \mathcal{M}_{3}
$$

where $i \mathcal{M}_{\text {eikonal }}, i \mathcal{M}_{1}, i \mathcal{M}_{2}$, and $i \mathcal{M}_{3}$ are given by Eqs. (3), (6), (20), and (23), respectively. This is our main result. We further recall [9] that one needs to use the covariant coupling in the large $x$ terms so that the above amplitude smoothly reduces to the eikonal amplitude $i \mathcal{M}_{\text {eikonal }}$ in the soft scattering limit, i.e., when $A\left(x^{+}, x^{-}, x_{t}\right) \rightarrow \not h S\left(x^{+}, x_{t}\right)$. To extract the quark propagator, one defines the effective vertex $\tau_{F}$ as

$$
i \mathcal{M}(p, \bar{q})=\bar{u}(\bar{q}) \tau_{F}(p, \bar{q}) u(p),
$$

in terms of which the propagator can be written as

$$
S_{F}(p, \bar{q})=(2 \pi)^{4} \delta^{4}(p-\bar{q}) S_{F}^{0}(p)+S_{F}^{0}(p) \tau_{F}(p, \bar{q}) S_{F}^{0}(\bar{q}) .
$$

A final remark is in order here: we have treated the target as consisting of gluons only and have totally ignored the contribution of quarks at large $x$. We expect sea quarks to appear when one performs a one-loop correction to our result and can therefore be included in principle. On the other hand, inclusion of valence quarks at large $x$ is an open problem at this point and will require a detailed investigation which is beyond the scope of this work.

\section{DISCUSSION AND SUMMARY}

We have derived the amplitude for scattering of a high energy quark on the gluon field of a proton or nucleus target including both small and large $x$ gluon modes of the target. This generalizes the standard expressions for eikonal scattering and is, to the best of our knowledge, the first gluon saturation-based calculation which includes large $x$ gluons (in the target proton or nucleus) as dynamical degrees of freedom. As such, it allows one to investigate many important high $p_{t}$ and/or large $x$ phenomena which are not accessible to the standard gluon saturation and color glass condensate formalism. This is specially essential for a proper and quantitative understanding of the outcome of the experiments at the proposed Electron Ion Collider and the Large Hadron Collider.

The derived scattering amplitude is a "tree-level" expression which can already be used to investigate several phenomena, for example, $p_{t}$ broadening and elastic energy loss as well as the nuclear modification factor $R_{p A}$ [13]. Due to the presence of large $x$ gluon fields in the target, the scattered quark can undergo an arbitrarily large deflection and pick up large transverse momenta. Furthermore, it can lose longitudinal momentum and undergo a potentially large rapidity loss which is not contained in saturation formalism. One can also extract the quark propagator from this scattering amplitude and use it to calculate the treelevel production cross sections for particle production in high energy collisions for any transverse momentum $p_{t}$ (at small or large $x$ ). One would also need to calculate the gluon propagator [14] in this formalism, which is a straightforward extension of the present work. This would allow one to investigate cold matter radiative energy loss including both the fully coherent (present in saturation formalism) as well as the partially coherent/incoherent energy loss which is not present in the saturation formalism. Examples of where our results in the present form can be used are single inclusive or dijet production in deep inelastic scattering as well as in high energy proton-proton and proton-nucleus collisions.

The present work generalizes the saturation formalism for tree-level processes and enlarges the transverse momentum range (recall $x$ and $p_{t}$ are kinematically related) where gluon saturation-based models are applied and improve their quantitative accuracy [15]. In addition, one would also be able to investigate forward-backward (in rapidity) correlations in our framework. Nevertheless, there is an important point worth emphasizing here. In the CGC formalism and for a large nucleus target, one can perform the color averaging procedure over the static target fields (or in a more commonly used terminology, over color charges $\rho$ ) using the McLerran-Venugopalan model [5]. This amounts to being able to calculate the initial conditions for rapidity evolution of physical observables, unlike the perturbative QCD case in which initial conditions for parton distribution functions are purely nonperturbative and thus parametrized. In our case, it is highly unlikely that one would be able to perform the averaging over the target gluon fields (or color charges) analytically and therefore would need to parametrize the initial conditions just like perturbative QCD. Also, there is the issue of matching the large $x$ expressions with that of small $x$ ones. Here, one can either follow the spirit of CGC formalism and impose a sharp boundary in $x$; for example, $x_{0}=0.01$ is commonly used in CGC formalism as the longitudinal momentum fraction below which CGC is applied. In this case, the new (noneikonal) terms in our expressions would be used for $x>x_{0}$ and the eikonal term for $x<x_{0}$. This is not an ideal approach but may be attempted as a first try in phenomenological applications. A less (kinematically) rigid approach could involve matching the small and large $x$ expressions at some value of the strength of the gluon field. For example, in CGC formalism, one considers gluon fields which are parametrically strong $O\left(\frac{1}{g}\right)$, whereas perturbative gluon fields are $O(g)$ so that one can choose a value of the coupling constant $g$ below which one takes the eikonal results and above which one takes the large $x$ expressions. This separation in either $x$ or $g$ would avoid double counting of target degrees of freedom. However, as pointed out above, one does not realistically expect to be able to compute, analytically, the expectation values of the operators involved in (the square of) our expressions. One would therefore need to parametrize the resulting expressions, which would then, similar to parton distribution functions in perturbative $\mathrm{QCD}$, serve as the 
initial conditions for the evolution (in $x$ and $Q^{2}$ ) dependence of our results, thereby avoiding the issue of double counting.

Naturally, one expects that our tree-level expression will be renormalized when one considers radiative (one-loop) corrections. In analogy with renormalization of product of Wilson lines [15-17] in small $x \mathrm{QCD}$, which leads to the Jalilian-Marian-Iancu-McLerran-Weigert-Leonidov-Kovner (JIMWLK)/Balitsky-Kovchegov evolution equations $[18,19]$, we expect the renormalization of the scattering cross section here to lead to a more general evolution equation which incorporates both the Dokshitzer-GribovLipatov-Altarelli-Parisi [20] and JIMWLK evolution equations; due to the presence of the large $x$ gluon field (not present in saturation formalism), one expects the one-loop corrections to result in the Dokshitzer-Gribov-LipatovAltarelli-Parisi evolution equation in the large $x$ limit. On the other hand and due to the presence of the eikonal term, one would expect to recover the JIMWLK/BalitskyKovchegov evolution equations in the small $x$ limit. Therefore, it will be enormously beneficial to calculate the one-loop corrections to our result. It may also be possible to reformulate this as an effective action approach, analogous to the McLerran-Venugopalan effective action [5]. If so, this would make it possible to treat both the early stages in the formation of a quark gluon plasma and the high $p_{t}$ jet energy loss in high energy heavy ion collisions using the same formalism, at least in the earliest times after the collision [21]. In summary, the present work takes the first step toward deriving a formalism that generalizes the color glass condensate framework by including the physics of high $p_{t}$ and large $x$.

\section{ACKNOWLEDGMENTS}

We acknowledge support from the U.S. DOE Office of Nuclear Physics through Grant No. DE-FG02-09ER41620 and from the Idex Paris-Saclay though a Jean d'Alembert grant. We would like to thank the staff of Pedro Pascual Science Center in Benasque, Spain, for their kind hospitality during the completion of this work. We also thank T. Altinoluk, N. Armesto, F. Gelis, E. Iancu, Yu. Kovchegov, A. Kovner, C. Lorcé, C. Marquet, A. H. Mueller, S. Munier, B. Pire, C. Salgado, G. Soyez, R. Venugopalan, D. Wertepny, and B. Xiao for critical questions, illuminating discussions, and helpful suggestions.
[1] R. Brock et al. (CTEQ Collaboration), Rev. Mod. Phys. 67, 157 (1995).

[2] E. Iancu and R. Venugopalan, arXiv:hep-ph/0303204; F. Gelis, E. Iancu, J. Jalilian-Marian, and R. Venugopalan, Annu. Rev. Nucl. Part. Sci. 60, 463 (2010); J. Jalilian-Marian and Y. V. Kovchegov, Prog. Part. Nucl. Phys. 56, 104 (2006); H. Weigert, Prog. Part. Nucl. Phys. 55, 461 (2005).

[3] L. V. Gribov, E. M. Levin, and M. G. Ryskin, Phys. Rep. 100, 1 (1983).

[4] A. H. Mueller and J. w. Qiu, Nucl. Phys. B268, 427 (1986).

[5] L. D. McLerran and R. Venugopalan, Phys. Rev. D 49, 2233 (1994); 49, 3352 (1994); 50, 2225 (1994); S. Jeon and R. Venugopalan, Phys. Rev. D 71, 125003 (2005); A. Dumitru, J. Jalilian-Marian, and E. Petreska, Phys. Rev. D 84, 014018 (2011).

[6] T. Lappi, EPJ Web Conf. 137, 07013 (2017).

[7] E. C. Aschenauer et al., arXiv:1602.03922.

[8] M. Hentschinski, A. Kusina, K. Kutak, and M. Serino, Eur. Phys. J. C 78, 174 (2018); M. Hentschinski, A. Kusina, and K. Kutak, Phys. Rev. D 94, 114013 (2016); O. Gituliar, M. Hentschinski, and K. Kutak, J. High Energy Phys. 01 (2016) 181; I. Balitsky and A. Tarasov, J. High Energy Phys. 06 (2016) 164; 10 (2015) 017.

[9] J. Jalilian-Marian, Phys. Rev. D 96, 074020 (2017).

[10] A. J. Baltz, F. Gelis, L. D. McLerran, and A. Peshier, Nucl. Phys. A695, 395 (2001); F. Gelis and A. Peshier, Nucl. Phys. A697, 879 (2002).

[11] A. Kovner and U. A. Wiedemann, arXiv:hep-ph/0304151; J. Casalderrey-Solana and C. A. Salgado, Acta Phys. Pol. B 38, 3731 (2007).
[12] T. Altinoluk and A. Dumitru, Phys. Rev. D 94, 074032 (2016); T. Altinoluk, N. Armesto, G. Beuf, and A. Moscoso, J. High Energy Phys. 01 (2016) 114; T. Altinoluk, N. Armesto, G. Beuf, M. Martínez, and C. A. Salgado, J. High Energy Phys. 07 (2014) 068; Y. V. Kovchegov and M. D. Sievert, arXiv:1808.09010.

[13] J. Jalilian-Marian, Nucl. Phys. A748, 664 (2005); J. JalilianMarian, Y. Nara, and R. Venugopalan, Phys. Lett. B 577, 54 (2003).

[14] A. Ayala, J. Jalilian-Marian, L. D. McLerran, and R. Venugopalan, Phys. Rev. D 52, 2935 (1995).

[15] G. A. Chirilli, B. W. Xiao, and F. Yuan, Phys. Rev. D 86, 054005 (2012); Phys. Rev. Lett. 108, 122301 (2012); B. W. Xiao and F. Yuan, arXiv:1407.6314; Z. B. Kang, I. Vitev, and H. Xing, Phys. Rev. Lett. 113, 062002 (2014); E. Iancu, A. H. Mueller, and D. N. Triantafyllopoulos, J. High Energy Phys. 12 (2016) 041; A. M. Stasto and D. Zaslavsky, Int. J. Mod. Phys. A 31, 1630039 (2016).

[16] A. Dumitru and J. Jalilian-Marian, Phys. Rev. Lett. 89, 022301 (2002); Phys. Lett. B 547, 15 (2002); A. Dumitru, A. Hayashigaki, and J. Jalilian-Marian, Nucl. Phys. A765, 464 (2006); A770, 57 (2006).

[17] A. Dumitru, J. Jalilian-Marian, T. Lappi, B. Schenke, and R. Venugopalan, Phys. Lett. B 706, 219 (2011); A. Dumitru and J. Jalilian-Marian, Phys. Rev. D 82, 074023 (2010).

[18] J. Jalilian-Marian, A. Kovner, L. D. McLerran, and H. Weigert, Phys. Rev. D 55, 5414 (1997); J. Jalilian-Marian, A. Kovner, A. Leonidov, and H. Weigert, Nucl. Phys. B504, 415 (1997); Phys. Rev. D 59, 014014 (1998); 59, 014015 (1998); 59, 034007 (1999); A. Kovner, J. G. Milhano, and 
H. Weigert, Phys. Rev. D 62, 114005 (2000); 61, 014012 (1999); E. Iancu, A. Leonidov, and L. D. McLerran, Nucl. Phys. A692, 583 (2001); Phys. Lett. B 510, 133 (2001); E. Ferreiro, E. Iancu, A. Leonidov, and L. McLerran, Nucl. Phys. A703, 489 (2002).

[19] I. Balitsky, Nucl. Phys. B463, 99 (1996); Y. V. Kovchegov, Phys. Rev. D 60, 034008 (1999).
[20] G. Altarelli and G. Parisi, Nucl. Phys. B126, 298 (1977); V. N. Gribov and L. N. Lipatov, Sov. J. Nucl. Phys. 15, 438 (1972); 15, 675 (1972); Yu. Dokshitzer, Sov. Phys. JETP 46, 641 (1977).

[21] A. Kovner, L. D. McLerran, and H. Weigert, Phys. Rev. D 52, 6231 (1995); 52, 3809 (1995). 